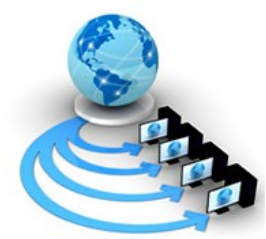

Volume 9, No. 6, November-December 2018

\title{
PARALLEL APPROACH OF VISUAL ACCESS TENDENCY FOR BIG DATA CLUSTER ASSESSMENT
}

\author{
Dr. Chukka Santhaiah \\ Department of computer science \& Engineering \\ Institute of Aeronautical Engineering. \\ Hyderabad, India
}

\author{
K. Sunanda* \\ Department of computer science \& Engineering \\ Institute of Aeronautical Engineering \\ Hyderabad, India
}

\begin{abstract}
Visual Access of Tendency (VAT) proficiency, for visually finding the amount of clusters in data. VAT makes a picture lattice work that can be made use of for visual examination of collecting disposition in either social or challenge data. A strategy is offered for ostensibly evaluating the celebration fondness of a course of action of Items $\mathrm{O}=\left\{\mathrm{o}_{1}, \mathrm{O}_{2}, \ldots \mathrm{o}_{\mathrm{n}}\right\}$ when we dealt with either as inquiry vectors or by numerical pair sensible difference concerns. Things are modified and the reordered framework of join sharp dissent inconsistencies is appeared as a power photo. Packages are shown by plain squares of pixels along one side. In endeavor we are suggesting identical method for aesthetic accessibility disposition for bundling to update the execution by revealing various datasets without a minute hold-up in the single display. The concern of choosing if packages are accessible as a stage preceding accredited gathering is called the examining of packing disposition. So below we are using parallel VAT to manage the issue. Rather than showing the masterminded individuality organizes as $2 \mathrm{D}$ dimensional degree photo to individual understanding as is concluded by VAT, we go after the alterations in diversity next corner to edge of the ODM. This examination is basic in recognizing the basic conjecture of VAT as well as VAT-based estimations and also, just much more typically, unique computations that trust, or like, Prim's Algorithm Based on this procedure we establish a Parallel Visual Gain access to of collecting Tendency (VAT) matter to analyze much getting to educational documents as well as demonstrate its central focuses the extent that complex nature as well as predisposition for utilization in a scattered figuring condition. Clusters are revealed by decrease squares of pixels along the edge to corner.
\end{abstract}

Keywords: VAT, clustering, Parallel Visual Access, ODM ,Data Visualization

\section{INTRODUCTION}

VAT winds up uncontrollable for significant educational buildups. The freshened VAT (reVAT) figuring lowers the amount of estimations finished by VAT, and also changes the photo link in a tactical plan of account outlines that are made use of for the aesthetic analysis action. In this way, reVAT beats the significant educational record issue which hinders VAT, but presents an additional issue: explanation of the course of action of reVAT account lays out winds up being astoundingly frustrating when the quantity of clusters is much reaching, or there is significant cover between get-togethers of points in the data. A structure is offered for clearly reviewing the affair tendency of a method of Objects $\mathrm{O}=\left\{\mathrm{o}_{1}, \mathrm{o}_{2}, . . \mathrm{o}_{\mathrm{n}}\right\}$ when they are often tended to either as difference vectors or by mathematical set smart aberration respects. The short articles are provided and also the reordered cross segment of suit sharp demand inconsistencies is turned up as a power picture. Groups are appeared by boring squares of pixels along the inclining. Nevertheless, in Existing structure we can generally process one dataset sometimes. In This paper we are recommending identical implementation or process for 2 datasets sometimes. In the identical VAT we are passing two significant differences question cross sections immediately as well as structure will certainly process them and reserve a couple of minutes. Choosing the proportion of events in a helpful hoarding is a vital issue in get-together examination. The Visual Access of (pack) Propensity (VAT) estimation is an inducing device for looking disposition, which passes on a hallmark photo of system as the depiction of complicated instructional buildups. Together, VAT can be computationally unbelievable for significant informing documents as a result of its $\mathrm{O}(\mathrm{N} 2)$ time varied high quality. This post recommends a rewarding parallel hope to breathe life right into the basic VAT.

We take into consideration a type of starter information examination pertaining to the version confirmation concern of gathering. Bunching or generating exam is the complication of limiting treatment of things $\mathrm{O}=$ into $\mathrm{c}$ identity-close departments due to open data and also some particularly depicted level of (gathering) resemblance. The kind of packs discovered is unquestionably pertaining to the belongings of the numerical version that inherent the party method. Every pressing check will certainly discover an optional (approximately $1 \leq \mathrm{c} \leq \mathrm{n}$ ) number of get-togethers, paying little respect to whether no "ensured" numbers exist. In this manner, each a basic sense basic thing to ask before using a specific (as well as potentially prejudicing) organizing count is: do packs exist utilizing all strategies? The problem of selecting if packs come as a stage prior to genuine amassing is known as the examining of get-together liking. Diverse formal (quantifiably based) and also pleasing frameworks for propensity evaluation are evaluated in Jain as well as Dubes as well as Everitt. Not any of today systems is definitely exceptional (or deplorable). The motivation driving these symbols is to intertwine a crucial and also normal visual method to handle the here and now gettogether of fondness evaluation mechanical social events. Perceptible slant for numerous facts evaluation snags have been all around researched over the most up to date twenty five years; Tukey as well as Cleveland [4] common hotspots some perceptible techniques. The perceptible approach for researching number propensity appeared below can be utilized in each of cases including 
mathematical data. It is valuable and also expected that advanced frameworks in gathering have a sharp phrase. As necessary we entitle this pristine instrument VAT (visual gain access to of tendency).

\section{RELATED WORK}

A framework is given for evidently checking the plan propensity of a course of action of Things $\mathrm{O}=\left\{\mathrm{o}_{1}, \mathrm{o}_{2}, . . \mathrm{o}_{\mathrm{n}}\right\}$ when they have a tendency to either as examinating vectors or by mathematical set wise uniqueness respects. The posts are swapped as well as the transformed setup of combine smart demand significant differences is turned up as a power photo. Numbers are appeared by reduce squares of pixels along the edge to edge. Another system has been given for vat making use of asked for individuality depiction. The propound requesting tally is homologous to Prim's estimate for locating the immaterial going across point tree of a weighted structure. The strategy can flag the distance of all over withdrew bunches by strategies for the proximity of dull squares of element on the conventional inclining of the ODI. This treatment appertains to all estimations and every last mathematical datum makes, finish or poor. A few 2 dimensional viewpoints recommend that ODI's might enable us "to glimpse" congruous possessions of focal request instructive papers. [2] Reliable VAT with time approach provide a starting assessment of time blueprint gathering with a thought on a novel outline-based level of identicalness, or, in other words consistent time relocation and also consistent ampleness scaling. Due to this measure a Visual Gain access to of pack Tendency (VAT) computation to contemplate enormous time course of action illuminating events and display its positive problems relatively as complex nature and also prejudice for implementation in a passed on enlisting condition. This computation is acknowledged as a cloud operation utilizing Flicker where the run-time of the giant flexible high quality difference network figuring's are minimized by up to 7.0 times in a 16 facility enrolling bundle with broadly higher promote aspects mean for even more noticeable preparation teams. This VAT figuring is affordable for use in Big Information settings where farthest point and also therapy of information is performed in a streamed preparing framework.

Masterminds under examination in Information Visualization are negligible globe systems. In this just how this metric can be battered through a brilliant training course of the system in context of semantic zooming is discussed. When the structure is spoiled right into a degrees of campaign of sub-deals with, a client can without a significant quantity of a stretch find events as well as subgroups of executing authorities and also understand their segments. Expanding Adjacency Matrix Traveler (ZAME), an acknowledgment tool for evaluating stories at a size of a broad number of concentrates as well as edges. Totals are handled into a pyramid placing that thinks about on-request paging to GPU shader tasks to assist plain multi range taking a look at. [5] Format mastermind problems are a specific class of combinatorial streamlining problems whose objective is to situate a timely arrangement of a data chart in such manner in which a specific target price is updated. This paper attempted to give an entire perspective of today cutting edge concerning strategies concerns. [6] Emphasis interface plots have as frequently as conceivable been made use of to deal with charts. In the layout attracting structure, different arrangements manage orchestrate systems consenting to rich requirements, for example, restricting the measure of edge-crossing focuses, engaging the degree in between the lengthiest side as well as the most minimal edge, and uncovering balances. We offer a gain access to looking two portrayals recollecting the genuine goal to demonstrate their specific focal shows as a strategy of nonexclusive assessment efforts. [7] A novel method is made use of via a solitary picture one can see all the collections. The framework depends upon a computation for low-capacity enclose of collected details, with the residential or commercial property that package between all events is made sure, paying little identification to their tendency. [8] We offer an electronic unsecured beginning contraption to help cross-castigating system serration with the running with goals: to look at changed framework phases, to find diagrams from the info, comment on it, as well as accumulate discovering. Seriating is a without supervision details mining procedure that reorders items right into a movement along a one-dimensional continuum to understand the whole strategy. Gathering administers posts to events, while serial names points to a problem inside an activity. [9] This calculation shows the results of examinations investigating the family member well worth (from a HCI point of view) of blueprint attracting feel as well as figuring's using a solitary overview. The end results exhibit that while some individual style influence human implementation, it is tough to share that one tally transcends to anything an additional from a social understanding viewpoint, Would certainly a close to stylish as well as mathematical close outcomes be relocating nearer on a plan of various describes, specific social implementation estimates, Would a similar cleaned as well as analytical family member results be particular with numerous social implementation estimates much more actions to this demand requirement to examined.

[10] Minimal world charts provide a technique to make versatile, instinctual representations of irrelevant globe outlines, involving the client to evaluate close to packs while prolong basic chart of the whole formation. The perception strategy uses a mix of both semantically as well as geometrical turns, while the plan is made by springtime fixed figuring utilizing a beginning late affected oblige to appear. [11] Info turning techniques is utilized for multivariate depiction of info. Especially, the consequences of a couple of exams recommend that 3-D representations can be a lot more disastrous than fixed shows in subject judgments of info events as well as information structure. [12] Instead of showing the cross area as a 2-dimensional lessen degree photo ODI for human understanding, VAT DT(Diagonal Tracing) isolates the framework by taking navels of different sorts along its edge to edge and hands down the disposition turns, with one of the most strong of them being the dcontort. [13] Framework Powering is discussed as, Offered a method of $\mathrm{n}$ focuses with a grid of pair wise resemblance matter, one ought to require to separate focuses into packs so essentially equivalent concentrates are as one and also particular ones disengaged. We offer an estimation calling for primarily organize managing that executes a very long time a little while later and also bears 
an abundant understanding like self-emphatic strolls around a chart. In this chart, the visual-mess problem in perceptions has been presented. Beginning there, we consider the cost-based, geometry-based, and also picture based edge-packaging method for structures, identical energizes, as well as stream maps.

\section{PROPOSED MODEL}

A methodology is offered for evidently considering the bundle inclination of objects when they have a tendency to either as inquiry vectors or by mathematical match sensible uniqueness areas. The write-ups are reordered as well as the reordered network of suit smart demand dissimilarities is showed up as a power image. Packs are turned up by decline squares of pixels along the corner to edge. Nevertheless, in Existing framework we can just refine one dataset sometimes. In VAT experience we are suggesting identical execution or process for 2 datasets sometimes. In the identical VAT we are passing two dissimilarities examine networks quickly and framework will certainly process them as well as set aside a few minutes.

Visual Access of Cluster Tendency for Vast Data Sets: Assessment of organizing affection is an essential start phase in put away assessment. One device for looking over pack propensity is the Visual Gain Access to Propensity calculation. VAT influences an image to sort out that can be used for visual gain access to of party propensity in either social or test information. Whatever considered VAT winds up un-restrain able for wide edifying get-togethers. The changed VAT (reVAT) figuring lessens the proportion of evaluations prepared by VAT, and also changes the photo random sample with a game-plan of account representations that are used for the aesthetic assessment step. Thus, reVAT massacres the excellent informing recap concern which hamper VAT, yet shows another problem: image of the technique of reVAT account charts swings to be especially troublesome when the percentage of packs is sweeping, or there is vital cover in between gatherings of articles in the data. In this paper, we recommend estimation called large VAT which (i) manage the wide data problem continued by VAT, and (ii) takes care of the understanding issue driven forward by reVAT. Big VAT blends the semi asking for method used by reVAT with a photo program of the strategy of account stories showing the squeezing disposition info with a VAT-like picture. A couple of numerical viewpoints are offered to deal with and support the brand-new structure. In the nuclear mix inquire about wizard, tools for data examination and also affirmation are essential for professionals to do take a look at job. Web Range is such a mechanical social event, or, to put it simply utilized in the assessment of EAST of Institute of Plasma Physics to exhibit waveforms as well as will be better for scientists to get to and also disengage data. It is an adjustment to the past device jScope and East Scope. Web Scope is a contraption with a B/S show up online for information exam and delineation on the EAST workplace, so it could be utilized via web program. With the target of getting to and also discovering the vital examination data on the web, this data evaluation and also monitoring framework is acknowledged as Java Applets tongue which can be signed up with right into a HTML web page. Thusly, authorities from all parts of the world can visit the tool WebScope with Net, or, to put it simply remarkable for them to different information at wherever as well as at whatever factor seeing that there is Internet, pointless to indicate it, moreover, it can show instructional document on different servers meanwhile. The greater bit of this isn't given by jScope and also EastScope. Thinking about, WebScope is an additional and also the entire all the in addition promising mechanical celebration for information assessment and instinct. This paper depicts the technique of its being displayed, its positive conditions, its synopsis and its request.

Tool for Visual Access of (Cluster) Tendency: A system is designated for ostensibly gauge the gathering aptness of a game plan of Objects $\mathrm{O}=\left\{\mathrm{o}_{1}, \mathrm{o}_{2}, . . \mathrm{o}_{\mathrm{n}}\right\}$ when they are addressed either as inquiry vectors or by numerical pair wise contrast regards. The things are flip-flop and the flipflop cross section of match keen dissent diversity is appeared as a power picture. Bundles are exhibited by dull squares of pixels along the corner to corner.

Visual Access of Cluster Tendency Using Diagonal Uncover: The aesthetic examination propensity system, for plainly discovering the measure of standard social affairs in information, made by J. C. Bezdek, R. J. Hathaway and J. M. Huband, is astonishingly important, at any rate there is area for overhauls. As opposed to showing the prepared disparity cross area (ODM) as a 2D lower degree picture for anthropoid translation as is ended up by VAT, we take after making the changes in significant differences further slanting of the ODM. This developments the 2D information framework (systems) into 1D shows, appeared as what we call the prejudice turns, which equips one to focus just on one inconstant, particularly the stature. One of these bends, called the dwind, comprehensibly displays the closeness of social occasion formation as factors of reference in tops and valleys, which can be managed human eyes as well as furthermore by the simulation. Our analytical tests presented that the simulation can get assemble frameworks from the d-contort also occasionally where the human eyes see no formation from the aesthetic yields of VAT. In addition, achievement on each analytical examination was attain utilizing the matching (resolved) technique of program criterion respects. Picking the action of packs in an edifying get-together is an essential problem in generate assessment. The Visual Accessibility of (pack) Tendency (VAT) calculation is an effective instrument for investigative regarding gathering disposition that makes a characteristic photo of framework as the representation of complicated edifying records. Regardless, VAT can be computationally over the top for vast helpful documents because of its $\mathrm{O}$ (N2) time diverse nature. In this paper, we propose a sensible parallel anticipate breathing life right into the central VAT.

\section{PRACTICAL RESULTS}

Practical Results deals with VAT process and Parallel VAT process of visual cluster representation. When the unordered dissimilarity matrix is given as input and the VAT, process the data given and displays the visual image of unordered dissimilarity matrix as ordered dissimilarity matrix. Parallel VAT deals with two data sets at a time which reduces the time in giving accurate results. The below Figure 1 illustrates the VAT image of dissimilarity matrix. 


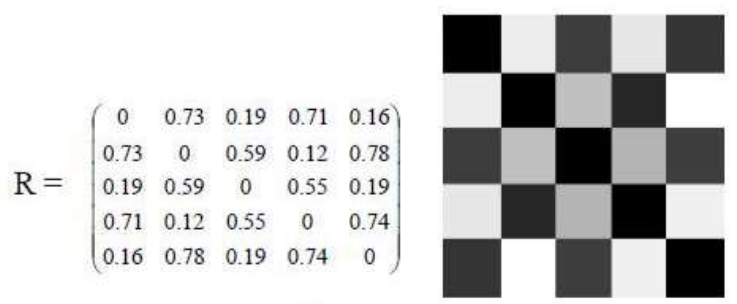

Fig. 1. A dissimilarity matrix and its corresponding dissimilarity image.

Parallel VAT Ordering and Display Algorithm:

Step 1 Set $\mathrm{K}=\{1,2, \ldots, \mathrm{n}\} ; \mathrm{I}=\mathrm{J}=\emptyset ; \mathrm{P}[0]=$ $(0, \ldots, 0)$. Step 2 Select $(i, j) € \operatorname{argmax}\{R \mathrm{Rpq}\}$ $\mathrm{p} € \mathrm{~K}, \mathrm{q} € \mathrm{~K}$

Set $P(1)=i ; I=\{i\}$; and $J=K-\{i\}$.

Step 3 For $r=2, \ldots, n$ :

Select $(i, j) €$ argmin $\{R p q\}$ p€I,q€J

Set $\mathrm{P}(\mathrm{r})=\mathrm{j}$; Replace $\mathrm{I} \leftarrow \mathrm{IU}\{\mathrm{j}\}$ and $\mathrm{J} \leftarrow \mathrm{J}-\{\mathrm{j}\}$.

Next $r$.

Step 4 Derive the system diversity matrix $\mathrm{R} \sim$ using the ordering array $\mathrm{P}$ as: $\mathrm{ij} \mathrm{R} \sim=\mathrm{RP}(\mathrm{i}) \mathrm{P}(\mathrm{j})$, for $1 \leq \mathrm{i}, \mathrm{j} \leq \mathrm{n}$.

Step 5 Display the interchange matrix $\mathrm{R} \sim$ as the ODII $\sim$ using the protocols given above.

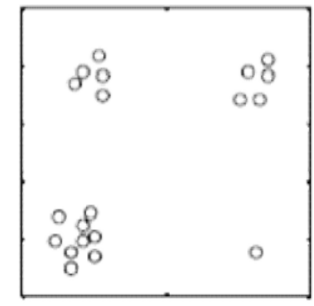

Fig. 2. Scatterolot of Data Set A.

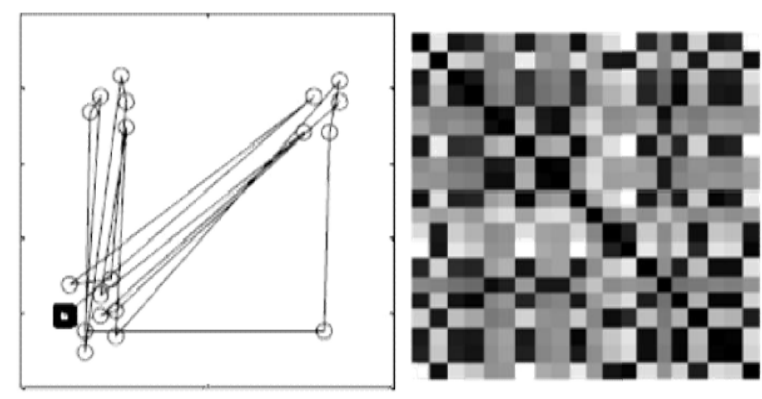

Fig. 3. Scatterplot and dissimilarity image for Data Set A (original random ordering)
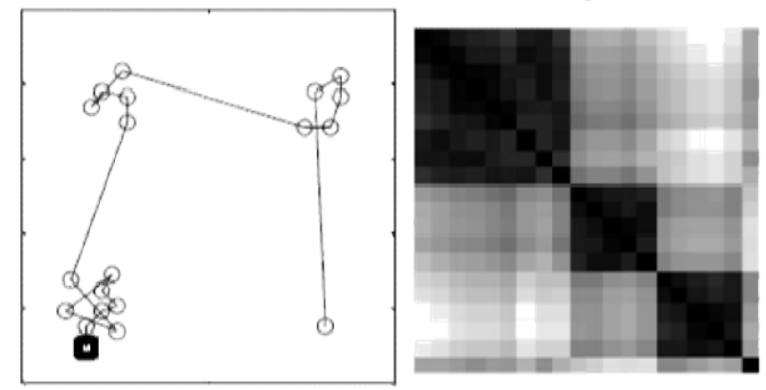

Fig. 4. Scatterplot and ODI for Data Set A (reordered)

\section{Abbreviations and Acronyms \\ VAT : Visual Access of (cluster)Tendency}

ODM : Ordered Dissimilarity Matrix

\section{CONCLUSION AND FUTURE ENHANCEMENT}

At last in this undertaking we showed that identical VAT will update the job capability by passing the two datasets at a possibility to framework. The structure will certainly become asked for cross location finally it will demonstrate the social affair partiality. We offered one more system for parallel apparently analyzing package disposition utilizing requested originality images. The proposed asking for count is connected to VAT figuring for discovering the unnecessary spreading over tree of a weighted graph. The method can signal the nearness of specifically separated events by approaches for the sign of boring squares of pixels on the essential corner to corner of the ODI. This method is suitable to all estimations and every single mathematical information kinds, total or not enough. A couple 2 dimensional models recommend that ODI's might equip us "to see" geometric homes of shrouded inquiry instructional lists. Here two varying datasets cross section can be in the meantime taken and visual examination of celebrations can be seen.

This parallel Visual Access Propensity supervises two datasets to demonstrate the packages evidently on the double. In the meantime we can offer estimations of 2 datasets as well as can see the aesthetic grouping. The future rise is to calls different datasets instantly can go to the structure.

\section{REFERENCES}

1. J.C. Bezdek Department of Computer Science, R.J. Hathaway Mathematics and Computer Science, VAT: A Tool for Visual Access of (Cluster) Tendency, 0-7803-7278-6/02/\$10.00 02002 IEEE.

2. Timothy B. Iredale, Sarah M. Erfani and Christopher Leckie, An Efficient Visual Access of Cluster Tendency Tool for Large-scale Time Series Data Sets. 978-1-5090-6034-4/17/\$31.00 c 2017 IEEE.

3. David Auber1LaBRI, Bordeaux, FranceYves Chiricota2Univ. Québec à Chicoutimi, CanadaFabien Jourdan, Guy Melançon LIRMM, Montpellier, France, Multiscale Visualization of Small World Networks.

4. https://www.researchgate.net/publication/221536265 , ZAME: Interactive Large-Scale Graph.

5. https://www.researchgate.net/publication/220, A Survey of Graph Layout Problems.

6. Mohammad Ghoniem ,Jean-Daniel Fekete ,Philippe Castagliola, On the readability of graphs using nodelink and matrix-based representations: controlled experiment and statistical analysis.

7. Yehuda Koren and David Harel, A Two-Way Visualization Method for Clustered Data.

8. Innar Liiv,1, Rain Opik,1 Jaan Ubi2 and John Stasko3, Visual matrix explorer for collaborative seriation.

9. H.C. Purchase The Department of Computer Science and Electrical Engineering, The University of Queensland, St. Lucia 4072, Qld, Australia, Effective information visualization: a study of graph drawing aesthetics and algorithms. 
10. Frank van Ham Jarke J. van Wijk, Interactive Visualization of Small World Graphs.[11].Selan Rodrigues dos Santos, A Framework for the

11. Visualization of Multidimensional and Multivariate Data.

12. Yingkang $\mathrm{Hu}$ Department of Mathematical Sciences, Georgia Southern University, Statesboro, USA, VATdt: Visual Access of Cluster Tendency Using Diagonal Tracing.
13. Hanson Zhou *M.I.T Computer Science and Artificial Intelligence Laboratory 200 Technology Square Cambridge, MA 02139, Clustering via Matrix Powering.

14. Hong Zhou_, Panpan $\mathrm{Xu}, \mathrm{Xiaoru} Y u a n$, and Huamin $\mathrm{Qu}$, Edge Bundling in Information Visualization.

15. Miklós Erdélyi, János Abonyi, Node Similarity-based Graph Clustering and Visualization. 